- Supporting Information -

\title{
Difficulties of Popular Density Functionals to Describe the Conformational Isomerism in Iodoacetic Acid
}

\author{
J. Philipp Wagner* \\ Institut für Organische Chemie, Eberhard Karls Universität Tübingen, Auf der Morgenstelle 18, \\ 72076 Tübingen \\ *Email: Philipp.wagner@orgchem.uni-tuebingen.de
}

Table of Contents

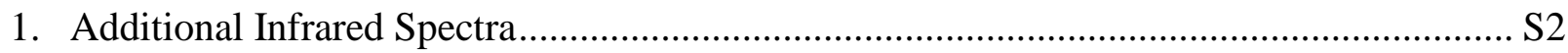

2. Additional Potential Energy Curves …………………................................................... S4

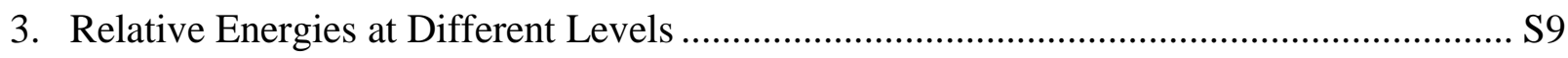

4. Electronic Energies at Different Levels ........................................................................ S11

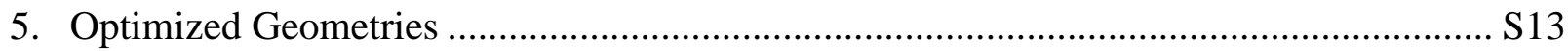

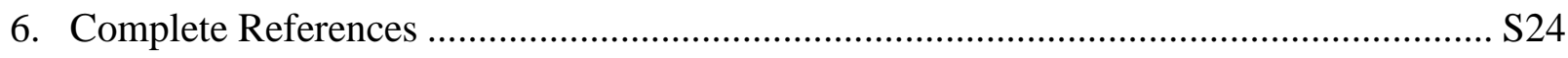




\section{Additional Infrared Spectra}

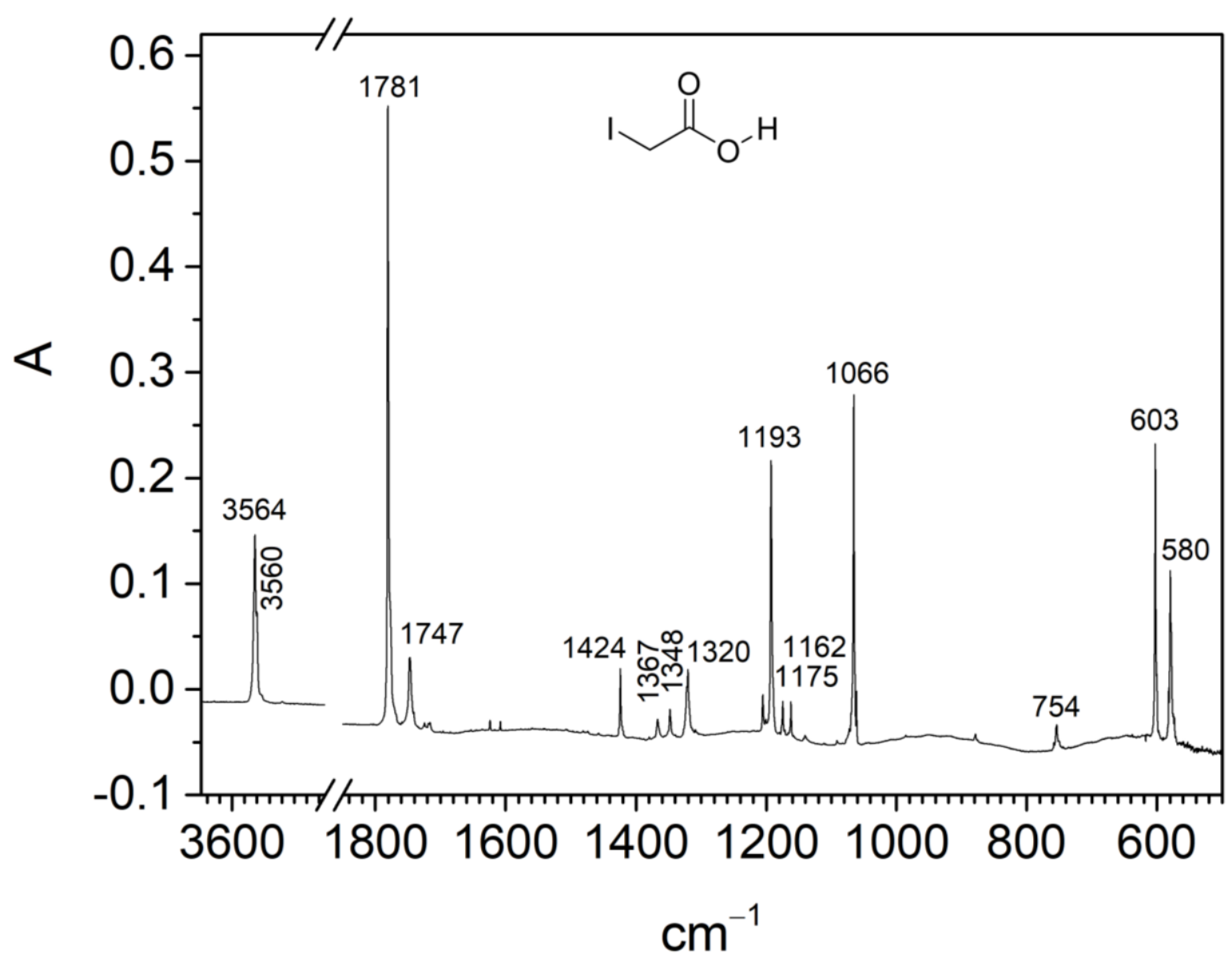

Figure S1. Infrared spectrum of matrix isolated iodoacetic acid in solid argon at $4 \mathrm{~K}$. 


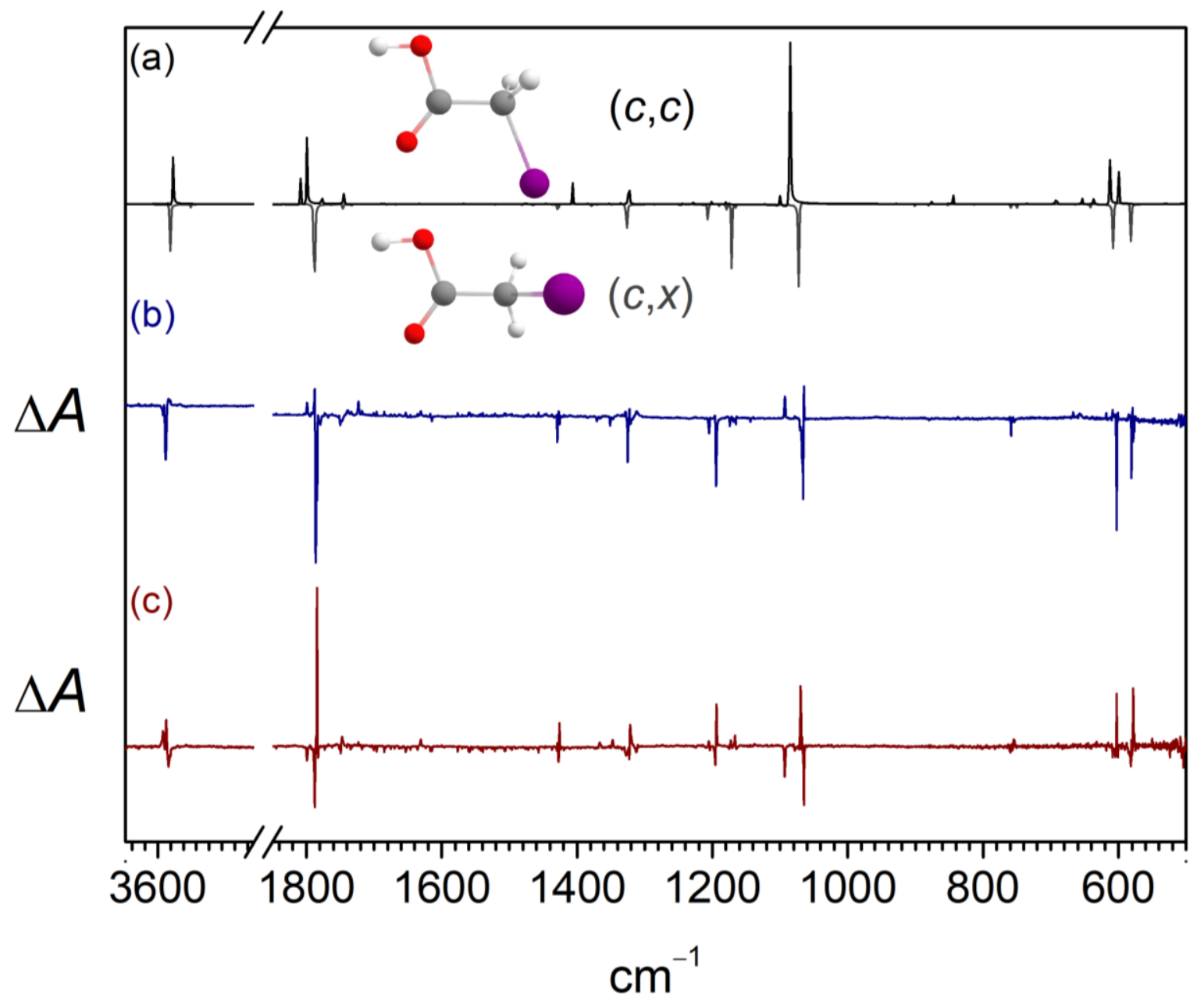

Figure S2. The top trace in (a) displays the anharmonic infrared spectra of conformers $(c, x)$ and $(c, c)$ pointing upwards and downwards, respectively, computed within the VPT2 approximation at the MP2/def2-TZVPP level of theory. The blue trace in (b) corresponds to the difference IR spectrum after UV irradiation (240-255 $\mathrm{nm}$ ) of a neon matrix doped with iodoacetic acid for 1.5 minutes. The red trace shows the difference after annealing of the irradiated matrix for ten minutes at $6.2 \mathrm{~K}$. 


\section{Additional Potential Energy Curves}

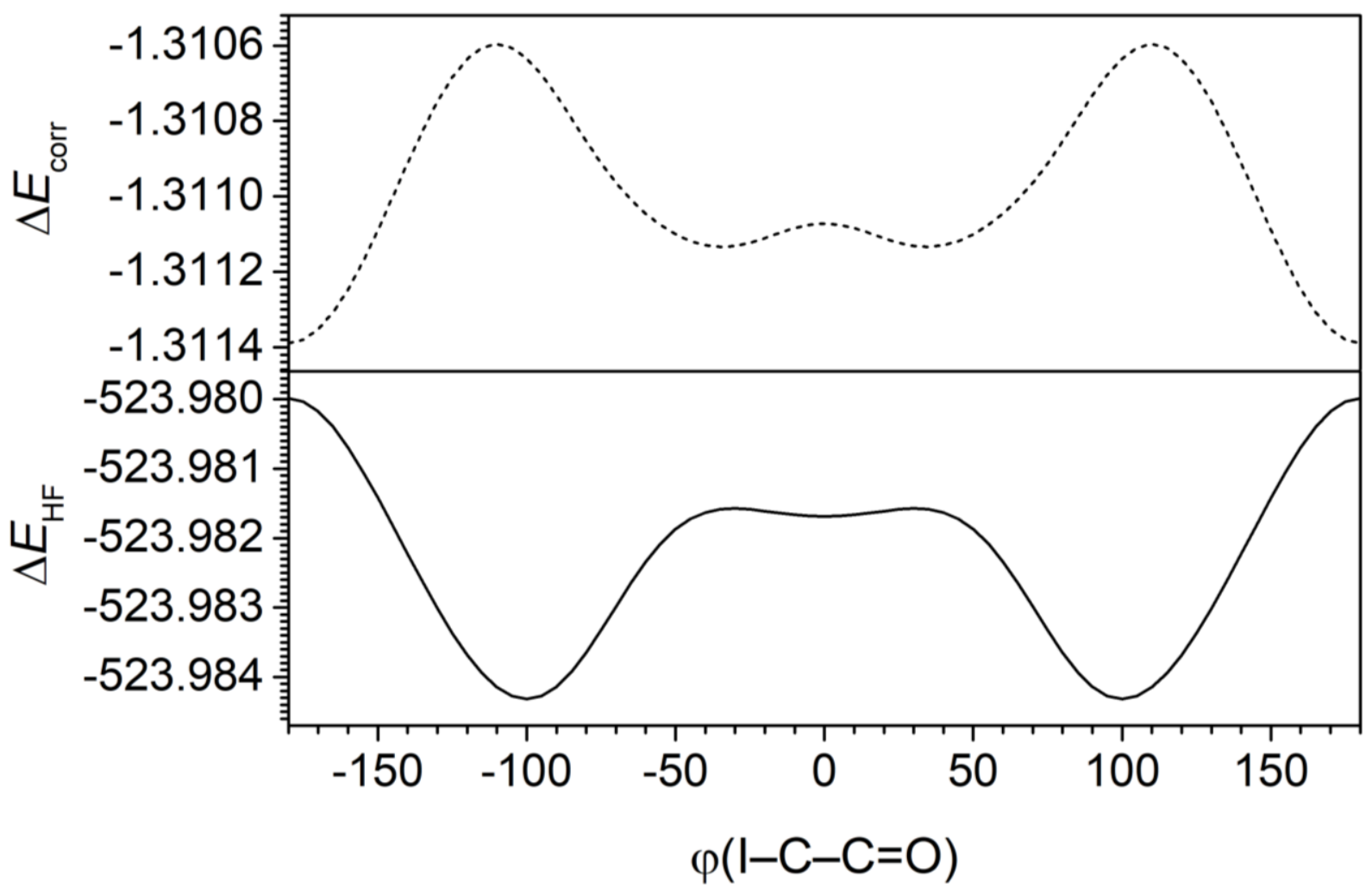

Figure S3. Absolute self-consistent field and correlation energies along the relaxed

CCSD(T)/def2-TZVPP//MP2/def2-TZVPP potential energy curves of $c i s$-iodoacetic acid. 


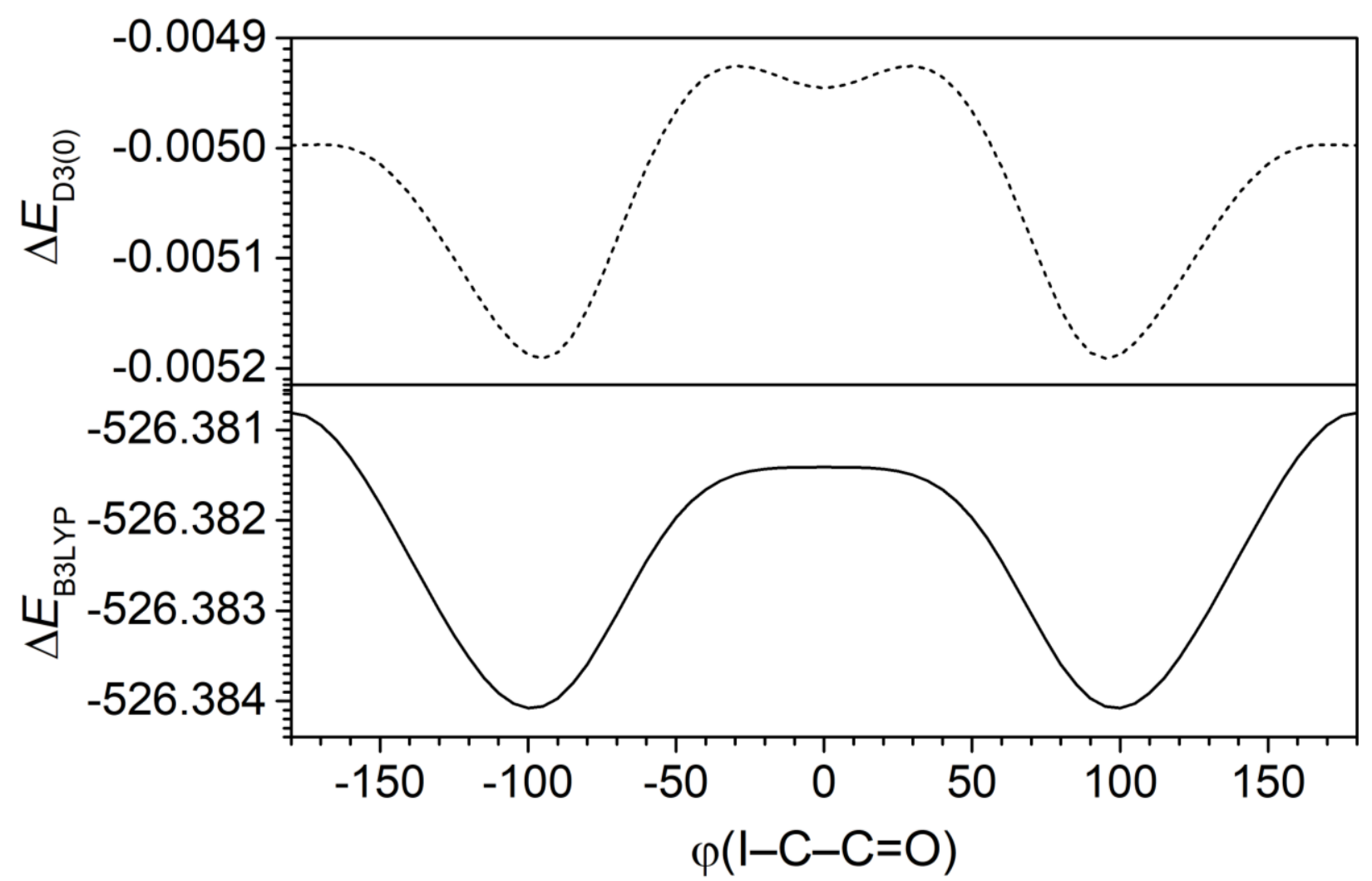

Figure S4. Absolute DFT electronic and D3(0)-dispersion correction energies along the relaxed B3LYP-D3(0)/def2-TZVPP potential energy curves of cis-iodoacetic acid. 


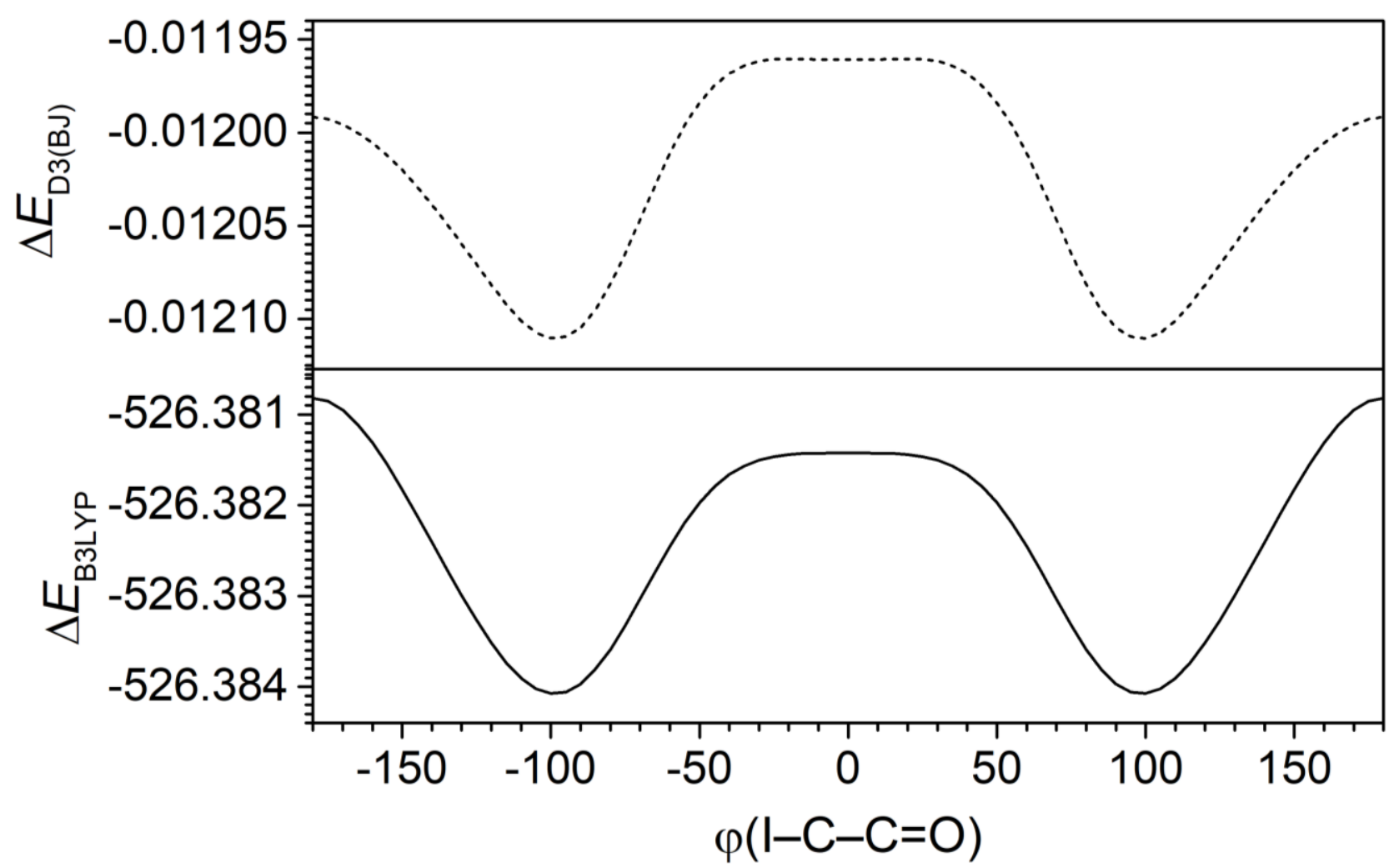

Figure S5. Absolute DFT electronic and D3(BJ)-dispersion correction energies along the relaxed B3LYP-D3(BJ)/def2-TZVPP potential energy curves of cis-iodoacetic acid. 


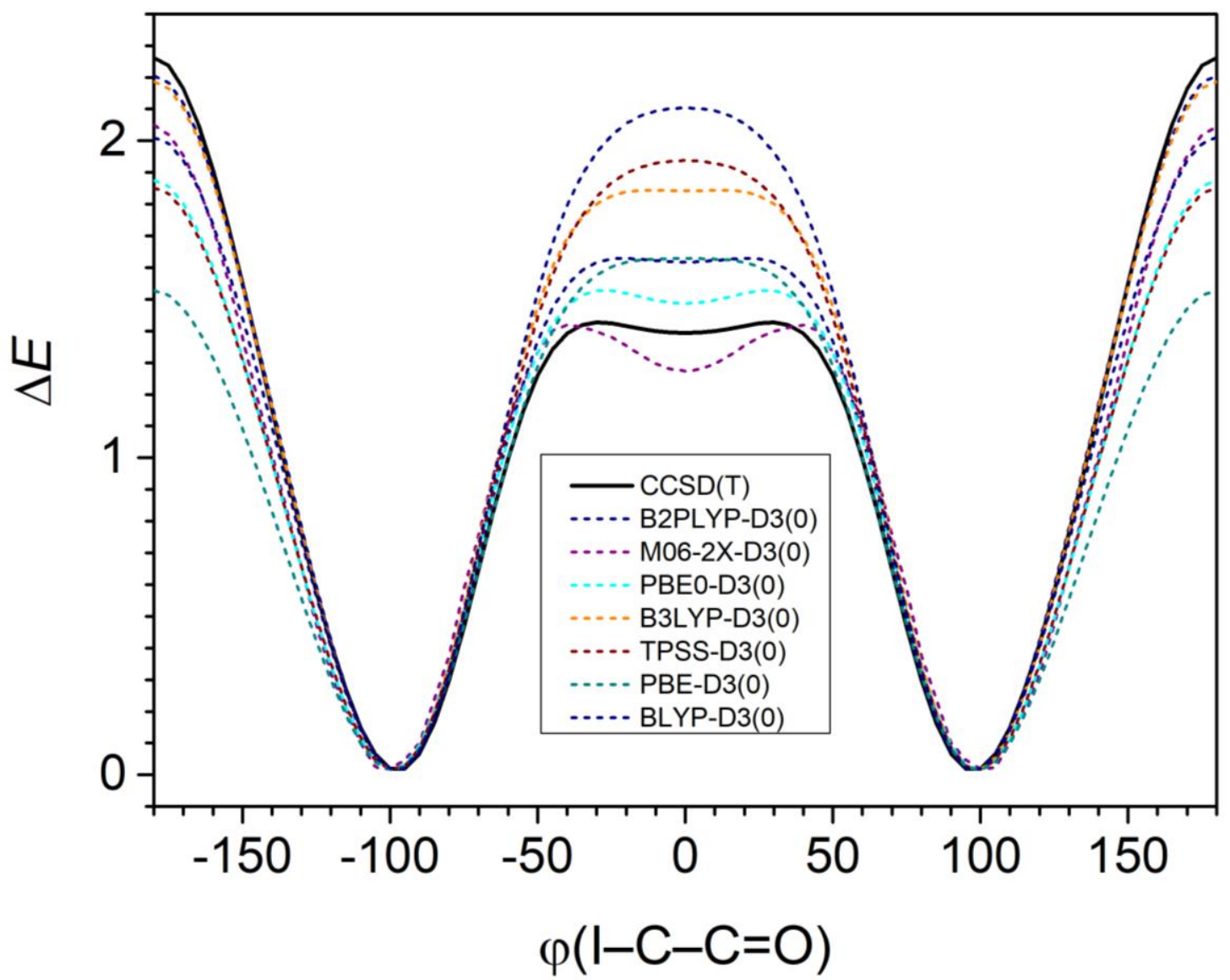

Figure S6. Plot drawn in analogy to Figure 5 in the main manuscript utilizing D3(0)-corrected DFAs. 


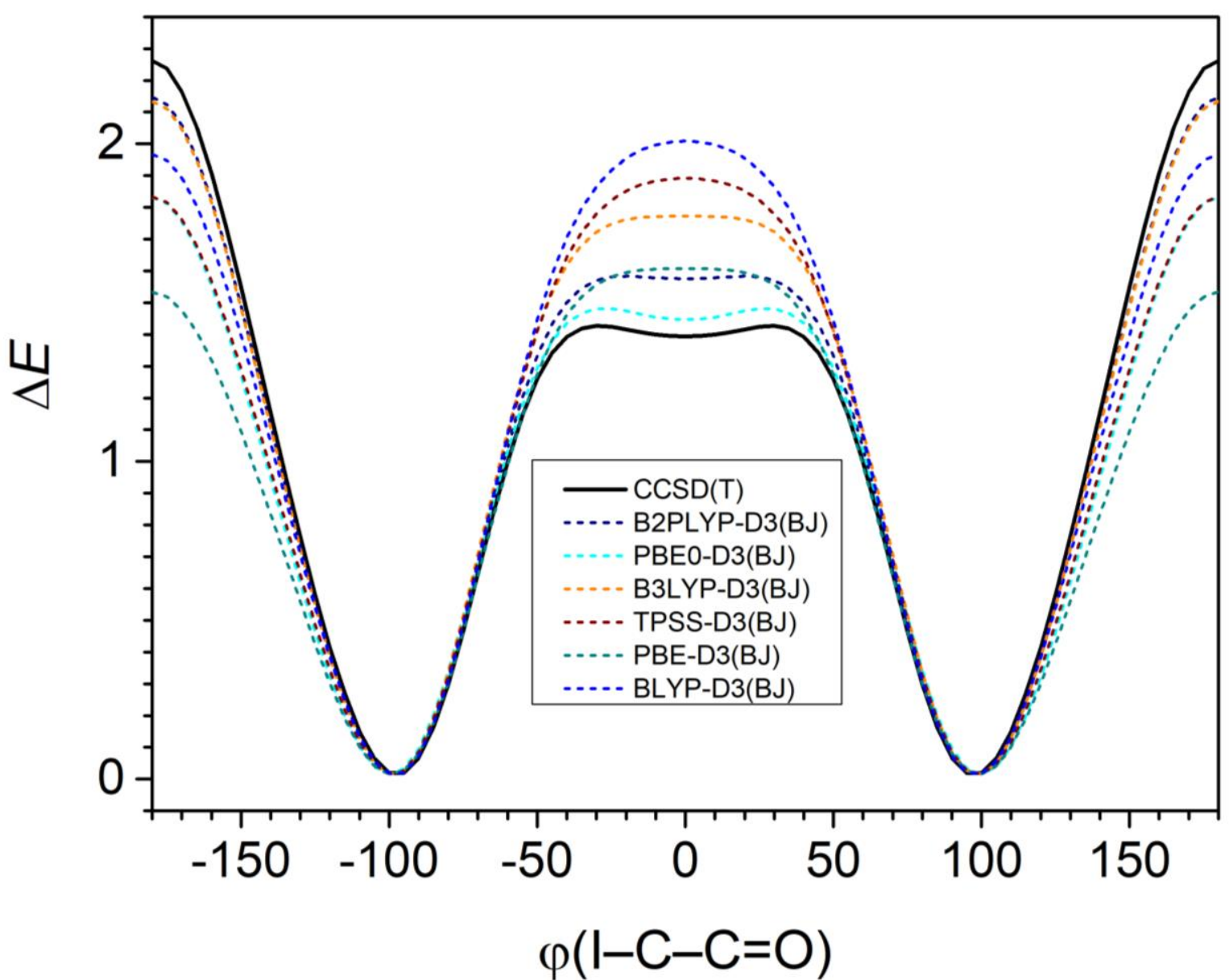

Figure S7. Plot drawn in analogy to Figure 5 in the main manuscript utilizing D3(BJ)-corrected DFAs. 


\section{Relative Energies at Different Levels}

Table S1. Relative electronic energies at different levels of theory utilizing a def2-QZVPP basis set are given in $\mathrm{kcal} \mathrm{mol}^{-1}$. The energies are computed employing MP2/def2-TZVPP geometries. For wave function methods, CBS extrapolated energies are reported.

\begin{tabular}{|c|c|c|c|c|c|c|}
\hline species & MP2 & $\mathrm{HF}$ & B2PLYP & B3LYP & BLYP & M06-2X \\
\hline$\left(c, x^{-}\right)$ & 0.00 & 0.00 & 0.00 & 0.00 & 0.00 & 0.00 \\
\hline$(c, c)$ & 1.25 & 1.63 & 1.48 & 1.66 & 1.82 & 1.27 \\
\hline$(c, \sigma)^{\ddagger}$ & 1.32 & 1.70 & 1.45 & 1.57 & 1.64 & 1.38 \\
\hline$(c, t)^{\ddagger}$ & 2.19 & 2.67 & 2.09 & 2.08 & 1.93 & 2.05 \\
\hline$(x, \xi)^{\ddagger}$ & 11.12 & 10.97 & 11.31 & 11.26 & 11.36 & 10.57 \\
\hline$(x, x)^{t}$ & 13.06 & 12.69 & 13.02 & 12.87 & 12.86 & 12.49 \\
\hline$\left(x, c^{-}\right)^{\ddagger}$ & 13.54 & 13.60 & 13.60 & 13.56 & 13.53 & 13.04 \\
\hline$\left(t, \beta^{-}\right)$ & 3.38 & 5.99 & 3.68 & 3.61 & 3.02 & 3.80 \\
\hline$(t, t)^{*}$ & 3.39 & 6.38 & 3.67 & 3.56 & 2.85 & 3.94 \\
\hline$(t, c)$ & 6.70 & 7.92 & 6.87 & 6.90 & 6.70 & 6.67 \\
\hline$\left(t, \sigma^{-}\right)^{\ddagger}$ & 7.14 & 8.21 & 7.12 & 7.05 & 6.73 & 7.16 \\
\hline species & PBE & PBE0 & TPSS & SCS-MP2 & BHandHLYP & B2GP-PLYP \\
\hline$\left(c, x^{-}\right)$ & 0.00 & 0.00 & 0.00 & 0.00 & 0.00 & 0.00 \\
\hline$(c, c)$ & 1.51 & 1.37 & 1.73 & 1.34 & 1.53 & 1.42 \\
\hline$(c, \sigma)^{\ddagger}$ & 1.43 & 1.39 & 1.59 & 1.38 & 1.55 & 1.43 \\
\hline$(c, t)^{t}$ & 1.54 & 1.80 & 1.79 & 2.33 & 2.27 & 2.13 \\
\hline$\left(x, \xi^{-}\right)^{\ddagger}$ & 11.48 & 11.32 & 11.46 & 10.96 & 11.13 & 11.29 \\
\hline$(x, x)^{\dagger}$ & 13.22 & 13.15 & 13.23 & 12.78 & 12.86 & 13.05 \\
\hline$\left(x, c^{-}\right)^{\ddagger}$ & 13.59 & 13.59 & 13.85 & 13.37 & 13.58 & 13.62 \\
\hline$\left(t, \beta^{-}\right)$ & 2.09 & 2.94 & 2.30 & 3.84 & 4.45 & 3.79 \\
\hline$(t, t)^{\ddagger}$ & 1.80 & 2.82 & 2.09 & 3.96 & 4.57 & 3.82 \\
\hline$(t, c)$ & 6.28 & 6.59 & 6.56 & 6.77 & 7.25 & 6.91 \\
\hline$\left(t, \sigma^{-}\right)^{*}$ & 6.50 & 6.91 & 6.68 & 7.14 & 7.53 & 7.22 \\
\hline species & $\begin{array}{c}\text { DSD- } \\
\text { PBEP86 }\end{array}$ & $\begin{array}{c}\text { CAM- } \\
\text { B3LYP }\end{array}$ & $\begin{array}{c}\text { B2PLYP- } \\
\text { D3 }\end{array}$ & B3LYP-D3 & BLYP-D3 & M06-2X-D3 \\
\hline$\left(c, x^{-}\right)$ & 0.00 & 0.00 & 0.00 & 0.00 & 0.00 & 0.00 \\
\hline$(c, c)$ & 1.30 & 1.24 & 1.61 & 1.88 & 2.10 & 1.26 \\
\hline$(c, \sigma)^{\ddagger}$ & 1.35 & 1.28 & 1.58 & 1.79 & 1.91 & 1.38 \\
\hline$(c, t)^{*}$ & 2.04 & 1.90 & 2.23 & 2.28 & 2.17 & 2.05 \\
\hline$\left(x, \xi^{-}\right)^{\ddagger}$ & 11.26 & 11.30 & 11.13 & 10.96 & 10.95 & 10.58 \\
\hline$(x, x)^{+}$ & 13.11 & 12.99 & 12.98 & 12.79 & 12.70 & 12.49 \\
\hline$\left(x, c^{-}\right)^{\ddagger}$ & 13.57 & 13.38 & 13.68 & 13.67 & 13.62 & 13.04 \\
\hline$\left(t, \beta^{-}\right)$ & 3.51 & 3.86 & 3.77 & 3.74 & 3.17 & 3.80 \\
\hline$(t, t)^{+}$ & 3.52 & 3.86 & 3.78 & 3.72 & 3.05 & 3.95 \\
\hline$(t, c)$ & 6.73 & 6.79 & 7.01 & 7.12 & 6.94 & 6.67 \\
\hline$\left(t, \sigma^{-}\right)^{\ddagger}$ & 7.11 & 7.09 & 7.27 & 7.27 & 6.96 & 7.17 \\
\hline
\end{tabular}




\begin{tabular}{lrrrrrr}
\hline species & PBE-D3 & PBE0-D3 & TPSS-D3 & $\begin{array}{r}\text { B2GP- } \\
\text { PLYP-D3 }\end{array}$ & wB97X-D3 & $\begin{array}{c}\text { B2PLYP- } \\
\text { D3(BJ) }\end{array}$ \\
\hline$\left(c, x^{-}\right)$ & 0.00 & 0.00 & 0.00 & 0.00 & 0.00 & 0.00 \\
$(c, c)$ & 1.60 & 1.49 & 1.88 & 1.52 & 1.16 & 1.54 \\
$(c, \sigma)^{\ddagger}$ & 1.52 & 1.51 & 1.74 & 1.53 & 1.24 & 1.51 \\
$(c, t)^{\ddagger}$ & 1.60 & 1.91 & 1.91 & 2.23 & 1.80 & 2.13 \\
$\left(x, \xi^{\ddagger}\right.$ & 11.34 & 11.16 & 11.22 & 11.15 & 11.25 & 11.18 \\
$(x, x)^{\ddagger}$ & 13.17 & 13.11 & 13.13 & 13.02 & 12.99 & 12.99 \\
$\left(x, c^{-}\right)^{\ddagger}$ & 13.61 & 13.65 & 13.88 & 13.68 & 13.34 & 13.63 \\
$\left(t, \beta^{-}\right)$ & 2.13 & 3.02 & 2.37 & 3.86 & 3.75 & 3.54 \\
$(t, t)^{\ddagger}$ & 1.85 & 2.91 & 2.19 & 3.90 & 3.74 & 3.54 \\
$(t, c)$ & 6.35 & 6.70 & 6.68 & 7.02 & 6.57 & 6.87 \\
$\left(t, \sigma^{-}\right)^{\ddagger}$ & 6.55 & 7.02 & 6.78 & 7.33 & 6.91 & 7.11 \\
\hline
\end{tabular}

\begin{tabular}{lrrrrrr}
\hline species & $\begin{array}{c}\text { B3LYP- } \\
\text { D3(BJ) }\end{array}$ & $\begin{array}{c}\text { BLYP- } \\
\text { D3(BJ) }\end{array}$ & $\begin{array}{c}\text { PBE- } \\
\text { D3(BJ) }\end{array}$ & $\begin{array}{c}\text { PBE0- } \\
\text { D3(BJ) }\end{array}$ & $\begin{array}{c}\text { TPSS- } \\
\text { D3(BJ) }\end{array}$ & $\begin{array}{c}\text { B2GP-PLYP- } \\
\text { D3(BJ) }\end{array}$ \\
\hline$\left(c, x^{-}\right)$ & 0.00 & 0.00 & 0.00 & 0.00 & 0.00 & 0.00 \\
$(c, c)$ & 1.76 & 1.95 & 1.57 & 1.44 & 1.82 & 1.45 \\
$(c, \sigma)^{\ddagger}$ & 1.68 & 1.76 & 1.49 & 1.46 & 1.68 & 1.47 \\
$(c, t)^{\ddagger}$ & 2.15 & 2.02 & 1.58 & 1.84 & 1.85 & 2.17 \\
$\left(x, \xi^{-}\right)^{\ddagger}$ & 10.99 & 11.03 & 11.34 & 11.20 & 11.28 & 11.18 \\
$(x, x)^{\ddagger}$ & 12.80 & 12.77 & 13.18 & 13.12 & 13.18 & 13.03 \\
$\left(x, c^{-}\right)^{\ddagger}$ & 13.61 & 13.60 & 13.62 & 13.64 & 13.90 & 13.65 \\
$\left(t, \beta^{-}\right)$ & 3.29 & 2.63 & 1.92 & 2.81 & 2.10 & 3.68 \\
$(t, t)^{\ddagger}$ & 3.26 & 2.48 & 1.64 & 2.70 & 1.91 & 3.71 \\
$(t, c)$ & 6.87 & 6.66 & 6.25 & 6.59 & 6.55 & 6.91 \\
$\left(t, \sigma^{-}\right)^{\ddagger}$ & 7.01 & 6.68 & 6.47 & 6.90 & 6.65 & 7.21 \\
\hline
\end{tabular}

\begin{tabular}{lrr}
\hline species & $\begin{array}{c}\text { BHandHLYP- } \\
\text { D3(BJ) }\end{array}$ & $\begin{array}{c}\text { DSD- } \\
\text { PBEP86- } \\
\text { D3(BJ) }\end{array}$ \\
\hline$\left(c, x^{-}\right)$ & 0.00 & 0.00 \\
$(c, c)$ & 1.60 & 1.34 \\
$(c, \sigma)^{\ddagger}$ & 1.63 & 1.38 \\
$\left(c, t^{\ddagger}\right.$ & 2.33 & 2.09 \\
$\left(x, \xi^{\ddagger}\right)^{\ddagger}$ & 10.92 & 11.14 \\
$\left(x, x^{\ddagger}\right.$ & 12.81 & 13.02 \\
$\left(x, c^{-}\right)^{\ddagger}$ & 13.63 & 13.54 \\
$\left(t, \beta^{-}\right)$ & 4.19 & 3.45 \\
$\left(t, t^{\ddagger}\right.$ & 4.33 & 3.48 \\
$(t, c)$ & 7.23 & 6.71 \\
$\left(t, \sigma^{-}\right)^{\ddagger}$ & 7.50 & 7.06 \\
\hline
\end{tabular}




\section{Electronic Energies at Different Levels}

Table S2. Electronic energies at different levels of theory utilizing a def2-QZVPP basis set are given in $E_{h}$. The energies are computed employing MP2/def2-TZVPP geometries. For wave function methods, CBS extrapolated energies are reported.

\begin{tabular}{lrrrrrr}
\hline species & MP2/CBS & HF/CBS & B2PLYP & B3LYP & BLYP & M06-2X \\
\hline$\left(c, x^{-}\right)$ & -525.682361 & -523.993973 & -526.040844 & -526.3984011 & -526.198359 & -526.1359463 \\
$(c, c)$ & -525.680376 & -523.991375 & -526.038489 & -526.3957594 & -526.195457 & -526.133926 \\
$(c, \sigma)^{\ddagger}$ & -525.680251 & -523.991268 & -526.038527 & -526.395893 & -526.195749 & -526.1337459 \\
$(c, t)^{\ddagger}$ & -525.678876 & -523.98972 & -526.037515 & -526.3950894 & -526.195286 & -526.1326828 \\
$\left(x, \xi^{-}\right)^{\dagger}$ & -525.664638 & -523.976488 & -526.022825 & -526.3804551 & -526.18025 & -526.1190979 \\
$(x, x)^{\dagger}$ & -525.661549 & -523.973745 & -526.020096 & -526.3778873 & -526.177872 & -526.1160422 \\
$\left(x, c^{-}\right)^{\dagger}$ & -525.660777 & -523.972304 & -526.019179 & -526.3767995 & -526.1768 & -526.1151674 \\
$\left(t, \beta^{-}\right)$ & -525.67698 & -523.984421 & -526.034986 & -526.3926546 & -526.193541 & -526.1298966 \\
$(t, t)^{\dagger}$ & -525.676957 & -523.983803 & -526.034996 & -526.3927267 & -526.193813 & -526.1296715 \\
$(t, c)$ & -525.671686 & -523.981355 & -526.029902 & -526.387398 & -526.187683 & -526.1253156 \\
$\left(t, \sigma^{-}\right)^{\dagger}$ & -525.670982 & -523.980883 & -526.029499 & -526.3871633 & -526.187633 & -526.1245343 \\
\hline
\end{tabular}

\begin{tabular}{|c|c|c|c|c|c|c|}
\hline species & PBE & PBE0 & TPSS & $\begin{array}{c}\text { B2PLYP- } \\
\text { D3 }\end{array}$ & B2LYP-D3 & BLYP-D3 \\
\hline$\left(c, x^{-}\right)$ & -526.039558 & -526.0629767 & -526.21849 & -526.043728 & -526.4035266 & -526.204459 \\
\hline$(c, c)$ & -526.037156 & -526.0607896 & -526.21574 & -526.041165 & -526.4005374 & -526.201108 \\
\hline$(c, \sigma)^{\ddagger}$ & -526.037275 & -526.0607584 & -526.215956 & -526.041203 & -526.4006707 & -526.201418 \\
\hline$(c, t)^{\ddagger}$ & -526.037099 & -526.0601069 & -526.215635 & -526.040181 & -526.3998919 & -526.201009 \\
\hline$\left(x, \xi^{-}\right)^{*}$ & -526.021265 & -526.0449446 & -526.200228 & -526.025996 & -526.3860645 & -526.187012 \\
\hline$(x, x)^{\dagger}$ & -526.01849 & -526.0420231 & -526.197411 & -526.023038 & -526.383139 & -526.184225 \\
\hline$\left(x, c^{-}\right)^{*}$ & -526.0179 & -526.0413123 & -526.196424 & -526.021935 & -526.381749 & -526.182757 \\
\hline$\left(t, \beta^{-}\right)$ & -526.036232 & -526.0582973 & -526.214824 & -526.037715 & -526.3975596 & -526.199402 \\
\hline$(t, t)^{\frac{1}{t}}$ & -526.036694 & -526.0584849 & -526.215155 & -526.037698 & -526.3975935 & -526.199603 \\
\hline$(t, c)$ & -526.029554 & -526.0524726 & -526.208035 & -526.032558 & -526.3921853 & -526.193392 \\
\hline$\left(t, \sigma^{-}\right)^{\ddagger}$ & -526.029202 & -526.0519664 & -526.207845 & -526.03214 & -526.3919484 & -526.193365 \\
\hline species & $\begin{array}{c}\text { M06-2X- } \\
\text { D3 } \\
\end{array}$ & PBE-D3 & PBE0-D3 & TPSS-D3 & $\begin{array}{c}\text { B2PLYP- } \\
\text { D3(BJ) }\end{array}$ & $\begin{array}{c}\text { B3LYP- } \\
\text { D3(BJ) }\end{array}$ \\
\hline$\left(c, x^{-}\right)$ & -526.1359844 & -526.042175 & -526.0659171 & -526.222414 & -526.046698 & -526.4106158 \\
\hline$(c, c)$ & -526.1339711 & -526.039627 & -526.0635447 & -526.219416 & -526.044251 & -526.4078055 \\
\hline$(c, \sigma)^{t}$ & -526.1337909 & -526.039751 & -526.0635115 & -526.219646 & -526.044292 & -526.4079426 \\
\hline$(c, t)^{*}$ & -526.1327224 & -526.039618 & -526.0628809 & -526.219377 & -526.043302 & -526.4071846 \\
\hline$\left(x, \xi^{-}\right)^{*}$ & -526.1191266 & -526.024096 & -526.0481284 & -526.204531 & -526.02888 & -526.3930974 \\
\hline$(x, x)^{\dagger}$ & -526.1160751 & -526.021194 & -526.0450313 & -526.201485 & -526.025999 & -526.3902114 \\
\hline$\left(x, c^{-}\right)^{\ddagger}$ & -526.1152049 & -526.020486 & -526.0441632 & -526.200287 & -526.024971 & -526.38892 \\
\hline$\left(t, \beta^{-}\right)$ & -526.1299221 & -526.038781 & -526.0611087 & -526.218639 & -526.04106 & -526.4053789 \\
\hline$(t, t)^{\frac{1}{t}}$ & -526.1296974 & -526.039228 & -526.0612815 & -526.218932 & -526.041056 & -526.4054224 \\
\hline$(t, c)$ & -526.1253476 & -526.032061 & -526.0552354 & -526.211761 & -526.035755 & -526.3996626 \\
\hline$\left(t, \sigma^{-}\right)^{\ddagger}$ & -526.1245645 & -526.031733 & -526.0547316 & -526.211603 & -526.035367 & -526.3994429 \\
\hline
\end{tabular}




\begin{tabular}{|c|c|c|c|c|c|c|}
\hline species & $\begin{array}{l}\text { BLYP- } \\
\text { D3(BJ) }\end{array}$ & $\begin{array}{c}\text { PBE- } \\
\text { D3(BJ) }\end{array}$ & $\begin{array}{l}\text { PBE0- } \\
\text { D3(BJ) }\end{array}$ & $\begin{array}{c}\text { TPSS- } \\
\text { D3(BJ) }\end{array}$ & $\mathrm{CCSD}(\mathrm{T}) / \mathrm{CBS}$ & $\begin{array}{c}\text { SCS- } \\
\mathrm{MP} 2 / \mathrm{CBS}\end{array}$ \\
\hline$\left(c, x^{-}\right)$ & -526.213249 & -526.046275 & -526.0689264 & -526.22715 & -525.6738776 & -525.579841 \\
\hline$(c, c)$ & -526.21014 & -526.043778 & -526.0666307 & -526.22425 & -525.6719561 & -525.577708 \\
\hline$(c, \sigma)^{\dagger}$ & -526.210437 & -526.043901 & -526.0666077 & -526.224475 & -525.6718470 & -525.577637 \\
\hline$(c, t)^{\dagger}$ & -526.210031 & -526.043757 & -526.0659938 & -526.224204 & -525.6705199 & -525.576136 \\
\hline$\left(x, \xi^{-}\right)^{\dagger}$ & -526.195667 & -526.028199 & -526.0510819 & -526.209176 & -525.6566853 & -525.562368 \\
\hline$(x, x)^{*}$ & -526.192897 & -526.025268 & -526.0480262 & -526.206151 & -525.6536287 & -525.559472 \\
\hline$\left(x, c^{-}\right)^{\dagger}$ & -526.191578 & -526.024572 & -526.0471959 & -526.204998 & -525.6529728 & -525.558542 \\
\hline$\left(t, \beta^{-}\right)$ & -526.209063 & -526.043211 & -526.0644452 & -526.223799 & -525.6684041 & -525.573716 \\
\hline$(t, t)^{t}$ & -526.209299 & -526.043658 & -526.0646186 & -526.224109 & -525.668301 & -525.573527 \\
\hline$(t, c)$ & -526.202642 & -526.036308 & -526.0584195 & -526.216708 & -525.6634118 & -525.569055 \\
\hline$\left(t, \sigma^{-}\right)^{t}$ & -526.202608 & -526.035966 & -526.0579373 & -526.216545 & -525.6627418 & -525.568466 \\
\hline species & BHandHLYP & $\begin{array}{l}\text { B2GP- } \\
\text { PLYP }\end{array}$ & $\begin{array}{c}\text { DSD- } \\
\text { PBEP86 }\end{array}$ & $\begin{array}{l}\text { CAM- } \\
\text { B3LYP }\end{array}$ & $\begin{array}{c}\text { B2GP- } \\
\text { PLYP-D3 }\end{array}$ & $\omega B 97 X-D 3$ \\
\hline$\left(c, x^{-}\right)$ & -526.1254845 & -525.973115 & -525.7614505 & -526.2164726 & -525.9752108 & -526.298022 \\
\hline$(c, c)$ & -526.1230495 & -525.970853 & -525.759385 & -526.2144995 & -525.9727942 & -526.296173 \\
\hline$(c, \sigma)^{\dagger}$ & -526.1230149 & -525.970833 & -525.7593009 & -526.2144393 & -525.9727745 & -526.296042 \\
\hline$(c, t)^{*}$ & -526.1218665 & -525.969718 & -525.7582023 & -526.213446 & -525.9716514 & -526.295147 \\
\hline$\left(x, \xi^{-}\right)^{\dagger}$ & -526.1077409 & -525.955126 & -525.7435051 & -526.1984711 & -525.9574435 & -526.28009 \\
\hline$(x, x)^{t}$ & -526.1049884 & -525.952316 & -525.7405589 & -526.1957751 & -525.9544566 & -526.277315 \\
\hline$\left(x, c^{-}\right)^{+}$ & -526.1038392 & -525.951413 & -525.7398206 & -526.1951484 & -525.9534126 & -526.27677 \\
\hline$\left(t, \beta^{-}\right)$ & -526.1183962 & -525.967068 & -525.7558571 & -526.2103232 & -525.969054 & -526.292043 \\
\hline$(t, t)^{\dagger}$ & -526.1181986 & -525.967025 & -525.7558475 & -526.2103198 & -525.9689889 & -526.292058 \\
\hline$(t, c)$ & -526.1139265 & -525.962102 & -525.7507261 & -526.2056575 & -525.9640274 & -526.287546 \\
\hline$\left(t, \sigma^{-}\right)^{t}$ & -526.1134926 & -525.961617 & -525.7501256 & -526.2051733 & -525.9635302 & -526.287014 \\
\hline
\end{tabular}

\begin{tabular}{|c|c|c|c|}
\hline species & $\begin{array}{l}\text { B2GP- } \\
\text { PLYP- } \\
\text { D3(BJ) }\end{array}$ & $\begin{array}{c}\text { BHandHLYP- } \\
\text { D3(BJ) }\end{array}$ & $\begin{array}{c}\text { DSD- } \\
\text { PBEP86- } \\
\text { D3(BJ) }\end{array}$ \\
\hline$\left(c, x^{-}\right)$ & -525.97819 & -526.135293 & -525.7122628 \\
\hline$(c, c)$ & -525.9758756 & -526.13274 & -525.7101228 \\
\hline$(c, \sigma)^{t}$ & -525.9758517 & -526.132703 & -525.7100571 \\
\hline$(c, t)^{t}$ & -525.9747294 & -526.13158 & -525.7089336 \\
\hline$\left(x, \xi^{-}\right)^{\dagger}$ & -525.9603725 & -526.117889 & -525.6945111 \\
\hline$(x, x)^{t}$ & -525.9574304 & -526.11488 & -525.6915184 \\
\hline$\left(x, c^{-}\right)^{\dagger}$ & -525.9564414 & -526.113574 & -525.6906922 \\
\hline$\left(t, \beta^{-}\right)$ & -525.9723284 & -526.128612 & -525.706762 \\
\hline$(t, t)^{\ddagger}$ & -525.9722724 & -526.128392 & -525.7067225 \\
\hline$(t, c)$ & -525.9671793 & -526.123771 & -525.7015734 \\
\hline$\left(t, \sigma^{-}\right)^{t}$ & -525.9666967 & -526.123343 & -525.7010058 \\
\hline
\end{tabular}




\section{Optimized Geometries}

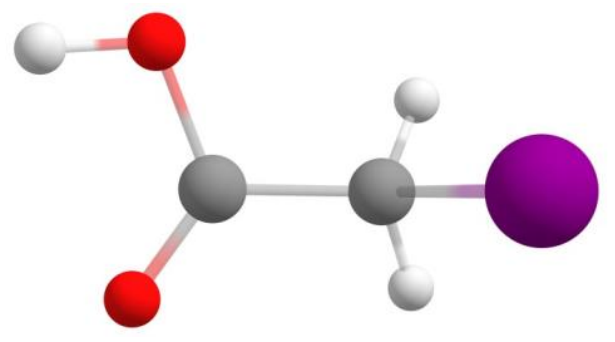

Figure S3. Optimized geometry of $\left(c, x^{-}\right)$(in $\AA$ ) at the MP2/def2-TZVPP level of theory.

$\begin{array}{lrrr}\mathrm{H} & 0.721085000 & 0.368172000 & 1.816017000 \\ \mathrm{O} & 2.216274000 & 1.180006000 & -0.012890000 \\ \mathrm{O} & 2.463037000 & -1.006079000 & -0.516095000 \\ \mathrm{C} & 1.882668000 & -0.126238000 & 0.072351000 \\ \mathrm{C} & 0.714499000 & -0.331240000 & 0.988889000 \\ \mathrm{H} & 0.683988000 & -1.360208000 & 1.321743000 \\ \mathrm{H} & 2.952641000 & 1.227295000 & -0.639629000 \\ \mathrm{I} & -1.082551000 & 0.021098000 & -0.087428000\end{array}$

Electronic MP2 energy (in $E_{\mathrm{h}}$ )

$-525.2695839$

Zero-point vibrational energy (in kcal $\mathrm{mol}^{-1}$ )

33.22916 


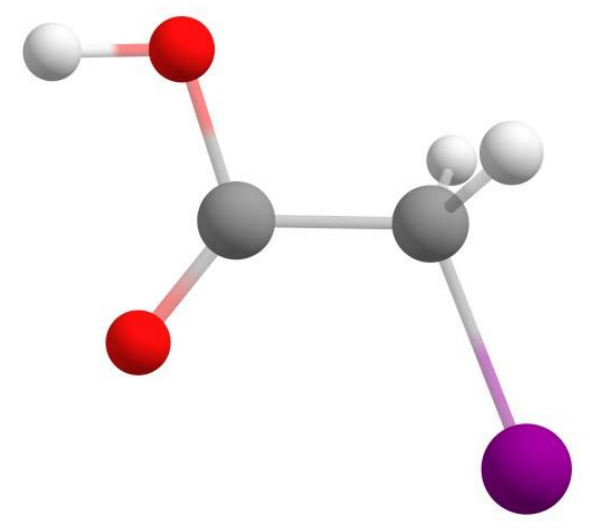

Figure S4. Optimized geometry of $(c, c)$ (in $\AA$ ) at the MP2/def2-TZVPP level of theory.

$\begin{array}{cccc}\mathrm{H} & -1.488679000 & -0.869487000 & 0.880198000 \\ \mathrm{O} & -0.489989000 & -3.076502000 & 0.000000000 \\ \mathrm{O} & 1.360610000 & -1.786273000 & 0.000000000 \\ \mathrm{C} & 0.162058000 & -1.887934000 & 0.000000000 \\ \mathrm{C} & -0.859820000 & -0.778803000 & 0.000000000 \\ \mathrm{H} & -1.488679000 & -0.869487000 & -0.880198000 \\ \mathrm{H} & 0.198965000 & -3.757026000 & 0.000000000 \\ \mathrm{I} & 0.000000000 & 1.139597000 & 0.000000000\end{array}$

Electronic MP2 energy (in $E_{\mathrm{h}}$ )

$-525.267412$

Zero-point vibrational energy (in $\mathrm{kcal} \mathrm{mol}^{-1}$ )

32.84198 


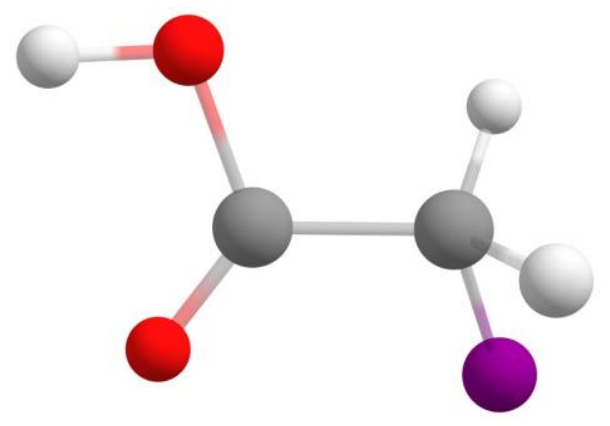

Figure S5. Optimized geometry of $(c, \sigma)^{\ddagger}($ in $\AA$ ) at the MP2/def2-TZVPP level of theory.

$\begin{array}{lrrr}\mathrm{H} & 0.767767000 & -1.044784000 & 1.442475000 \\ \mathrm{O} & 2.979534000 & -0.592323000 & -0.294521000 \\ \mathrm{O} & 1.874658000 & 1.320681000 & 0.159964000 \\ \mathrm{C} & 1.886546000 & 0.120544000 & 0.068984000 \\ \mathrm{C} & 0.752458000 & -0.822609000 & 0.378883000 \\ \mathrm{H} & 0.850813000 & -1.743721000 & -0.182123000 \\ \mathrm{H} & 3.685379000 & 0.057247000 & -0.430034000 \\ \mathrm{I} & -1.131538000 & 0.021071000 & -0.046058000\end{array}$

Electronic MP2 energy (in $E_{\mathrm{h}}$ )

$-525.2673125$

Zero-point vibrational energy (in kcal mol ${ }^{-1}$ )

32.94115 


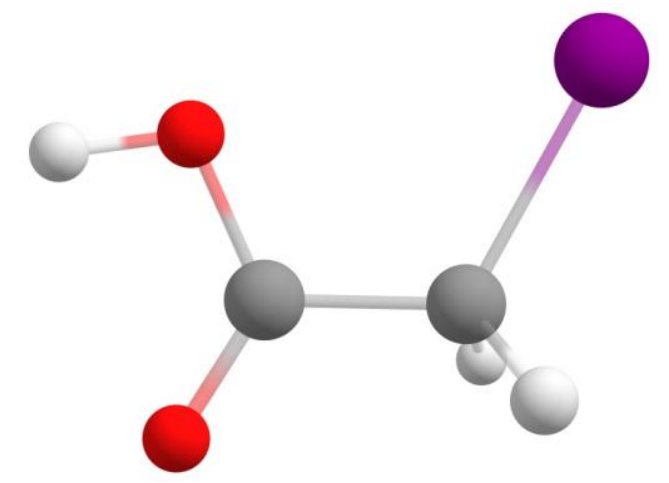

Figure S6. Optimized geometry of $(c, t)^{\ddagger}$ (in $\AA$ ) at the MP2/def2-TZVPP level of theory.

$\begin{array}{lrrr}\text { H } & -1.539169000 & -0.841277000 & 0.878987000 \\ \text { O } & 1.310651000 & -1.726095000 & 0.000000000 \\ \text { O } & -0.462452000 & -3.111791000 & 0.000000000 \\ \text { C } & -0.004388000 & -1.993378000 & 0.000000000 \\ \text { C } & -0.905569000 & -0.783013000 & 0.000000000 \\ \text { H } & -1.539169000 & -0.841277000 & -0.878987000 \\ \text { H } & 1.752483000 & -2.588539000 & 0.000000000 \\ \text { I } & 0.000000000 & 1.125142000 & 0.000000000\end{array}$

Electronic MP2 energy (in $\left.E_{\mathrm{h}}\right)$

$-525.2659252$

Zero-point vibrational energy (in kcal mol${ }^{-1}$ )

32.94459 


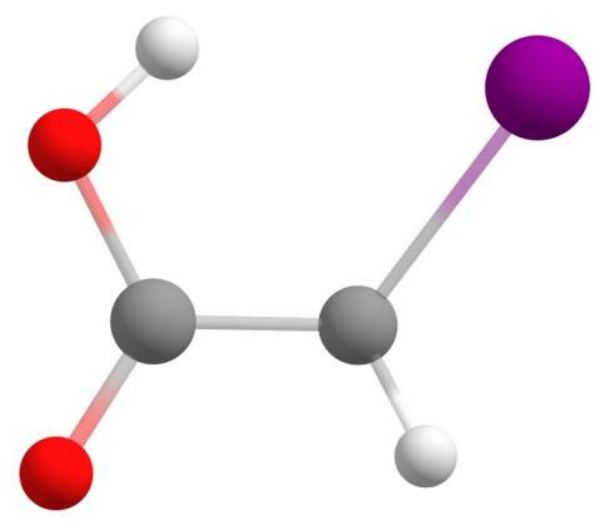

Figure S7. Optimized geometry of $\left(t, \beta^{-}\right)$(in $\AA$ ) at the MP2/def2-TZVPP level of theory.

$\begin{array}{lrrr}\mathrm{H} & 0.837021000 & 1.785161000 & 0.286426000 \\ \mathrm{O} & 1.876148000 & -1.306661000 & -0.114086000 \\ \mathrm{O} & 3.049647000 & 0.545304000 & 0.267515000 \\ \mathrm{C} & 1.998147000 & 0.025611000 & -0.002836000 \\ \mathrm{C} & 0.784257000 & 0.884932000 & -0.312558000 \\ \mathrm{H} & 0.803328000 & 1.147132000 & -1.366820000 \\ \mathrm{H} & 0.943059000 & -1.526950000 & -0.253007000 \\ \mathrm{I} & -1.107249000 & -0.014675000 & 0.037704000\end{array}$

Electronic MP2 energy (in $E_{\mathrm{h}}$ )

$-525.2635142$

Zero-point vibrational energy (in $\mathrm{kcal} \mathrm{mol}^{-1}$ )

32.91336 


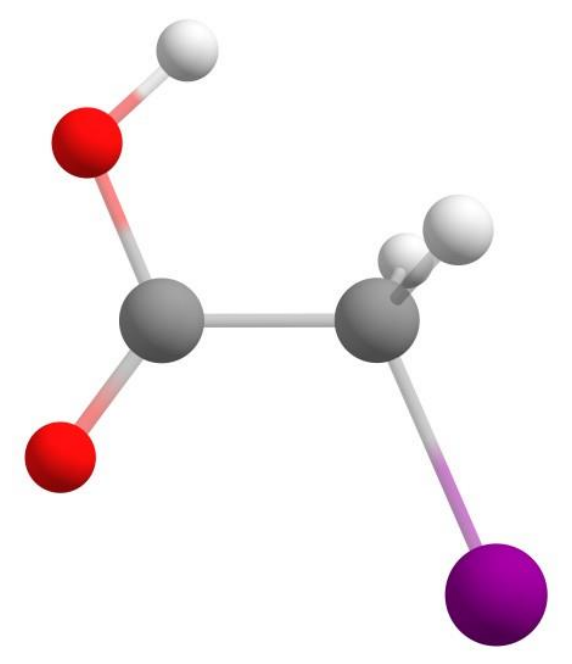

Figure S8. Optimized geometry of $(t, c)$ (in $\AA$ ) at the MP2/def2-TZVPP level of theory.

$\begin{array}{llll}\mathrm{H} & -1.469404000 & -0.873995000 & 0.884141000 \\ \mathrm{O} & -0.364410000 & -3.140632000 & 0.000000000 \\ \mathrm{O} & 1.383391000 & -1.764388000 & 0.000000000 \\ \mathrm{C} & 0.194030000 & -1.899184000 & 0.000000000 \\ \mathrm{C} & -0.842023000 & -0.790420000 & 0.000000000 \\ \mathrm{H} & -1.469404000 & -0.873995000 & -0.884141000 \\ \mathrm{H} & -1.325079000 & -3.067796000 & 0.000000000 \\ \mathrm{I} & 0.000000000 & 1.135728000 & 0.000000000\end{array}$

Electronic MP2 energy (in $\left.E_{\mathrm{h}}\right)$

$-525.2584894$

Zero-point vibrational energy (in kcal mol ${ }^{-1}$ )

32.68043 


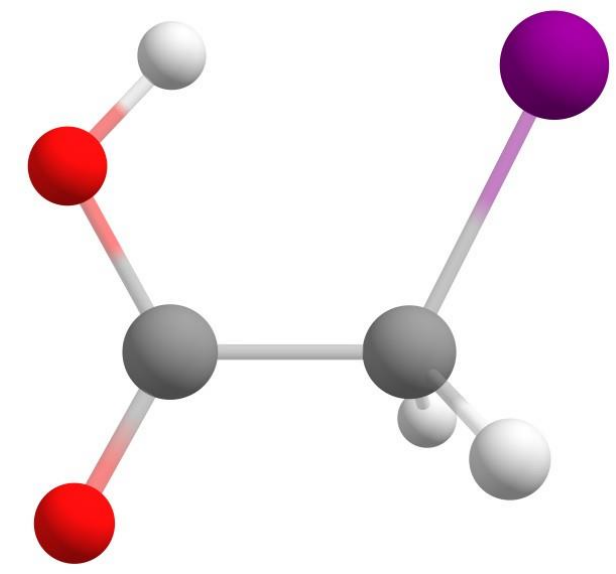

Figure S9. Optimized geometry of $(t, t)^{\ddagger}$ (in $\AA$ ) at the MP2/def2-TZVPP level of theory.

$\begin{array}{lrrc}\mathrm{H} & 0.837056000 & -1.547197000 & 0.882941000 \\ \mathrm{O} & 1.846138000 & 1.316213000 & 0.000060000 \\ \mathrm{O} & 3.109556000 & -0.515961000 & -0.000058000 \\ \mathrm{C} & 2.016268000 & -0.011891000 & 0.000012000 \\ \mathrm{C} & 0.792613000 & -0.918932000 & 0.000074000 \\ \mathrm{H} & 0.837076000 & -1.547343000 & -0.882687000 \\ \mathrm{H} & 0.896227000 & 1.51182000 & 0.000095000 \\ \mathrm{I} & -1.114513000 & 0.014458000 & -0.000016000\end{array}$

Electronic MP2 energy (in $E_{\mathrm{h}}$ )

$-525.263416$

Zero-point vibrational energy (in $\mathrm{kcal} \mathrm{mol}^{-1}$ )

32.82862 


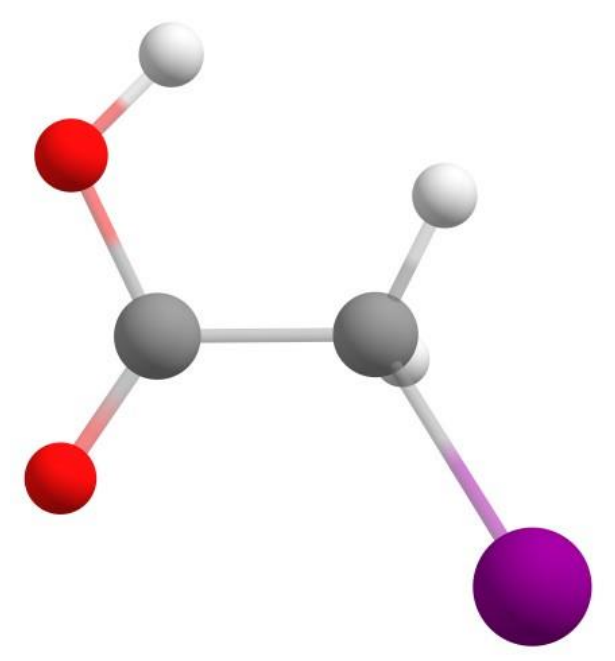

Figure S10. Optimized geometry of $\left(t, \sigma^{-}\right)^{t}$ (in $\AA$ ) at the MP2/def2-TZVPP level of theory.

$\begin{array}{lrrr}1 & -0.758528000 & 0.776310000 & -1.613729000 \\ 8 & -2.989778000 & 0.500388000 & 0.388210000 \\ 8 & -1.888486000 & -1.353459000 & -0.154499000 \\ 6 & -1.895769000 & -0.157600000 & -0.076306000 \\ 6 & -0.761468000 & 0.743402000 & -0.527747000 \\ 1 & -0.833300000 & 1.752861000 & -0.135786000 \\ 1 & -2.818439000 & 1.447321000 & 0.403969000 \\ 53 & 1.120374000 & -0.012580000 & 0.058494000\end{array}$

Electronic MP2 energy (in $E_{\mathrm{h}}$ )

$-525.2579132$

Zero-point vibrational energy (in $\mathrm{kcal} \mathrm{mol}^{-1}$ )

32.8189 


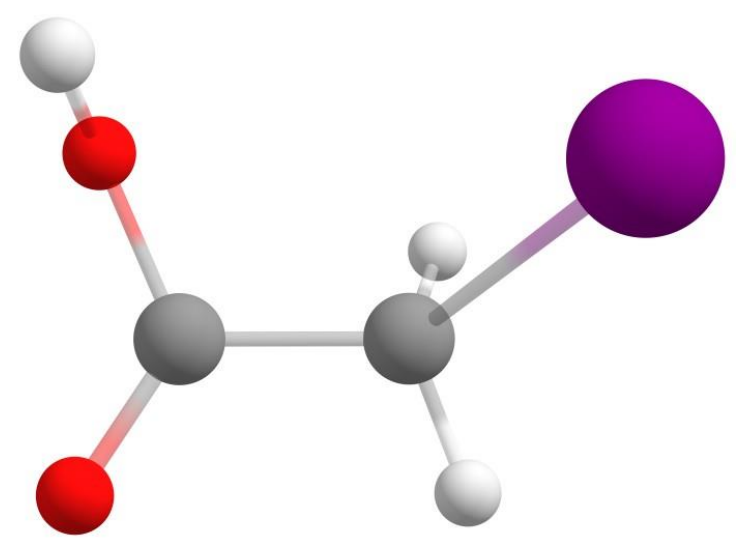

Figure S11. Optimized geometry of $\left(x, \xi^{-}\right)^{\ddagger}$ (in $\AA$ ) at the MP2/def2-TZVPP level of theory.

$\begin{array}{lrrr}\mathrm{H} & 0.731266000 & -0.236010000 & 1.839643000 \\ \mathrm{O} & 2.098224000 & 1.243440000 & 0.200368000 \\ \mathrm{O} & 2.622107000 & -0.802393000 & -0.620408000 \\ \mathrm{C} & 1.894390000 & -0.110698000 & 0.037441000 \\ \mathrm{C} & 0.736787000 & -0.640468000 & 0.833903000 \\ \mathrm{H} & 0.723496000 & -1.722470000 & 0.822277000 \\ \mathrm{H} & 1.597406000 & 1.727576000 & -0.464791000 \\ \mathrm{I} & -1.067960000 & 0.022821000 & -0.076696000\end{array}$

Electronic MP2 energy (in $E_{\mathrm{h}}$ )

$-525.2514413$

Zero-point vibrational energy (in kcal mol${ }^{-1}$ )

32.0786 


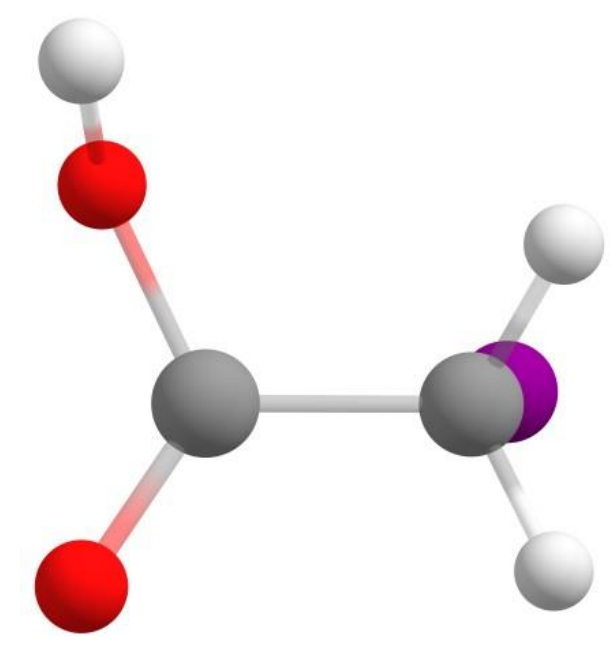

Figure S12. Optimized geometry of $(x, x)^{\ddagger}$ (in $\AA$ ) at the MP2/def2-TZVPP level of theory.

$\begin{array}{llll}\mathrm{H} & -0.730566000 & -0.183539000 & -1.835500000 \\ \mathrm{O} & -2.135035000 & -1.220772000 & 0.114498000 \\ \mathrm{O} & -2.512846000 & 0.968976000 & 0.546618000 \\ \mathrm{C} & -1.889592000 & 0.129696000 & -0.042834000 \\ \mathrm{C} & -0.727772000 & 0.435008000 & -0.944828000 \\ \mathrm{H} & -0.700367000 & 1.490733000 & -1.182110000 \\ \mathrm{H} & -2.717592000 & -1.527018000 & -0.588081000 \\ \mathrm{I} & 1.076146000 & -0.021774000 & 0.080052000\end{array}$

Electronic MP2 energy (in $\left.E_{\mathrm{h}}\right)$

$-525.2485335$

Zero-point vibrational energy (in $\mathrm{kcal} \mathrm{mol}^{-1}$ )

32.05899 


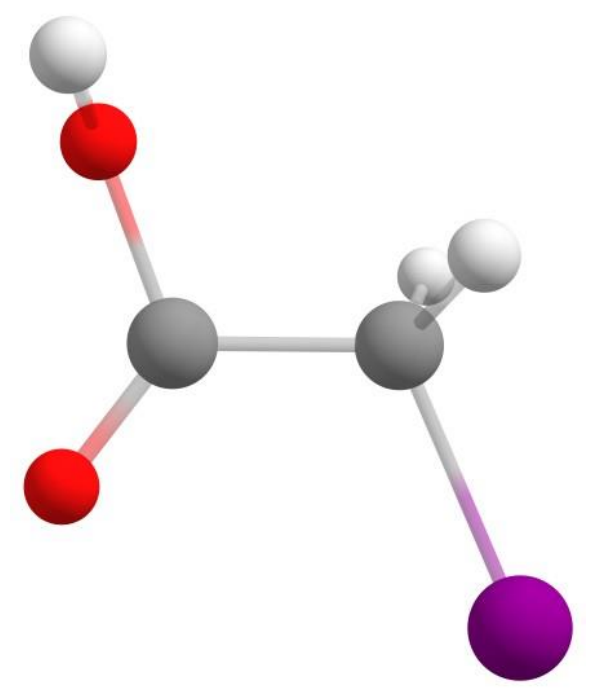

Figure S13. Optimized geometry of $\left(x, c^{-}\right)^{\sharp}$ (in $\AA$ ) at the MP2/def2-TZVPP level of theory.

$\begin{array}{lrrr}\mathrm{H} & 0.872669000 & -1.534525000 & 0.768053000 \\ \mathrm{O} & 3.129267000 & -0.473524000 & -0.060856000 \\ \mathrm{O} & 1.798444000 & 1.359083000 & -0.013995000 \\ \mathrm{C} & 1.900043000 & 0.168590000 & -0.011498000 \\ \mathrm{C} & 0.779412000 & -0.840312000 & -0.062425000 \\ \mathrm{H} & 0.868819000 & -1.408129000 & -0.984258000 \\ \mathrm{H} & 3.405000000 & -0.715928000 & 0.829329000 \\ \mathrm{I} & -1.144244000 & 0.011404000 & 0.008099000\end{array}$

Electronic MP2 energy (in $E_{\mathrm{h}}$ )

$-525.2476135$

Zero-point vibrational energy (in $\mathrm{kcal} \mathrm{mol}^{-1}$ )

31.7280 


\section{Complete References}

Gaussian reference:

Frisch, M. J.; Trucks, G. W.; Schlegel, H. B.; E.Scuseria, G.; Robb, M. A.; Cheeseman, J. R.; Scalmani, G.; Barone, V.; Mennucci, B.; Petersson, G. A.; Nakatsuji, H.; Caricato, M.; Li, X.; Hratchian, H. P.; Izmaylov, A. F.; Bloino, J.; Zheng, G.; Sonnenberg, J. L.; Hada, M.; Ehara, M.; Toyota, K.; Fukuda, R.; Hasegawa, J.; Ishida, M.; Nakajima, T.; Honda, Y.; Kitao, O.; Nakai, H.; Vreven, T.; Montgomery, J. A.; Peralta, J. E.; Ogliaro, F.; Bearpark, M.; Heyd, J. J.; Brothers, E.; Kudin, K. N.; Staroverov, V. N.; Kobayashi, R.; Normand, J.; Raghavachari, K.; Rendell, A.; Burant, J. C.; Iyengar, S. S.; Tomasi, J.; Cossi, M.; Rega, N.; Millam, J. M.; Klene, M.; Knox, J. E.; Cross, J. B.; Bakken, V.; Adamo, C.; Jaramillo, J.; Gomperts, R.; Stratmann, R. E.; Yazyev, O.; Austin, A. J.; Cammi, R.; Pomelli, C.; Ochterski, J. W.; Martin, R. L.; Morokuma, K.; Zakrzewski, V. G.; Voth, G. A.; Salvador, P.; Dannenberg, J. J.; Dapprich, S.; Daniels, A. D.; Farkas, Ö.;

Foresman, J. B.; Ortiz, J. V.; Cioslowski, J.; Fox, D. J., Gaussian 09, Revision D.01 \& Gaussian 16, Revision C.01, Gaussian, Inc., Wallingford CT, 2009. 